\title{
The electronic structure, dielectric properties and infrared vibrational spectrum of fayalite: an ab initio simulation with an all-electron Gaussian basis set and the B3LYP functional
}

\author{
Y. Noël ${ }^{\mathrm{a}}$, M. De La Pierre ${ }^{\mathrm{b}}$, L. Maschio ${ }^{\mathrm{b}}$, M. Rérat ${ }^{\mathrm{c}}$, C.M. Zicovich-Wilson ${ }^{\mathrm{d}}$, R. \\ Dovesi $^{\mathrm{b}}$ \\ ${ }^{a}$ Institut des Sciences de la Terre de Paris, (UMR 7193 UPMC-CNRS), UPMC - Paris Universitas, France \\ ${ }^{b}$ Dipartimento di Chimica IFM, Università di Torino and NIS - Nanostructured Interfaces and Surfaces - \\ Centre of Excellence, http://www.nis.unito.it, Via P. Giuria 7, 10125 Torino, Italy \\ ${ }^{c}$ Equipe de Chimie Physique, IPREM UMR5254, Université de Pau et des Pays de l'Adour, 64000 Pau, \\ France \\ ${ }^{d}$ Facultad de Ciencias, Universidad Autónoma del Estado de Morelos, Av. Universidad, 1001, Col. \\ Chamilpa, 62209 Cuernavaca (Morelos), Mexico
}

\begin{abstract}
The electronic structure, the static and high frequency dielectric tensors and the infrared spectrum of fayalite $\mathrm{Fe}_{2} \mathrm{SiO}_{4}$, the Fe-rich end-member of olivine solid solutions, are explored at an ab initio quantum mechanical level, by using an all-electron Gaussian type basis set, the B3LYP hybrid DFT functional and the CRYSTAL09 code. Mulliken population analysis and spin density maps illustrate the electronic structure, characterized by a nearly pure $\mathrm{d}^{6}$, high-spin configuration of the transition metal atom. The full set of IR wavenumbers and intensities is computed. The availability of highly accurate synchrotron radiation data (Suto et al. (2002) $A \& A, 389: 568)$ permits a very accurate comparison between simulated and measured quantities, in primis wavenumbers $(v)$ and oscillator strengths $(f)$. The mean absolute difference $\overline{|\Delta v|}$ is as small as $4 \mathrm{~cm}^{-1}$, and the maximum absolute difference $\left|\Delta v_{\text {max }}\right|$ never exceeds $12 \mathrm{~cm}^{-1}$, whereas the summed absolute difference $\Delta F$ between $f^{\text {exp }}$ and $f^{\text {calc }}$ is around $10 \%$. Modes not detected in the experiment turn out to be (i) characterized by low computed intensity, or (ii) very close to a large intense peak. Computed and experimental IR reflectance curves are in striking agreement, too. The nature of the vibrational modes is investi-
\end{abstract}

Email address: roberto.dovesi@unito.it (R. Dovesi) 
gated by means of isotopic substitutions, which clarify the participation of the various atomic species to each mode.

\section{Introduction}

Olivines are important rock-forming silicates, as they belong to the most abundant phases of the Earth's crust and upper mantle [1]; they represent a relevant component of interplanetary and interstellar solid particles [2], too.

Olivine solid solutions and the two pure end-members, $\mathrm{Mg}_{2} \mathrm{SiO}_{4}$ forsterite and $\mathrm{Fe}_{2} \mathrm{SiO}_{4}$ fayalite, have been the subject of many experimental investigations concerning their crystallographic $[3,4,5]$, thermodynamic $[5,6]$ and spectroscopic properties. Concerning this last aspect, transmission, reflection and absorption infrared spectra have been collected over the years $[5,7,8,9,10,11,12,13]$; the characterization of the spectrum is however not complete, probably due to degeneracies, low intensity of some fundamentals, and presence of overtones in the fundamental region.

This class of compounds represents a stimulating case study for modern periodic quantum mechanical codes, due to the relatively large unit cell (28 atoms), high symmetry, presence of transition metal atoms. In a previous work by some of the present authors [14], IR and Raman active modes of forsterite, i.e. the Mg-rich end-member, were investigated at the ab initio level. The agreement with the available experimental data was excellent.

In the present paper, we extend our investigation to the other end-member, fayalite, whose $\mathrm{Fe}^{2+}$ ion in $\mathrm{d}^{6}$, high-spin configuration is responsible for a Jahn-Teller distortion of the octahedral site, requiring a fine modeling of the electronic density. The comparison of the IR spectrum with experiments is particularly significant for this compound, thanks to the availability of accurate reflectance spectra, obtained by using the Japanese infrared beamline of the synchrotron radiation facility SPring8 [12].

Recently implemented computational tools are applied in this study, allowing a complete characterization of the vibrational properties, beyond the simulation of the full set of infrared frequencies: the $\mathrm{CPHF} / \mathrm{KS}$ method for the calculation of the optical dielectric tensor [15, 16], the Berry Phase scheme to compute IR intensities [17], the 
automated construction of the reflectance spectrum for the all the crystallographic directions, according to a classical damped harmonic oscillator model, directly from the parameters obtained at the ab initio level [18]. Additional information include the characterization of the $\mathrm{Fe}$ ions spin state and the analysis of the nature of the vibrational modes in the different spectral regions (the latter by means of isotopic substitution and graphical animation of the modes).

To the authors' knowledge this is the first ab initio calculation of the vibrational properties of fayalite: previous ab initio studies were limited to the discussion of the structural and ground-state electronic properties either at ambient conditions or at high pressures $[19,20,21]$.

The paper is structured as follows: Section 2 deals with the adopted computational method; in Section 3 results are reported and commented for the structure, the electronic configuration and the infrared properties; Section 4 presents the main conclusions.

\section{Computational details}

Calculations were performed with the CRYSTAL09 periodic ab initio code [22], by using an all-electron Gaussian type basis set and the hybrid B3LYP functional $[23,24,25]$, that has recently been shown to provide excellent results for geometry and vibrational frequencies of forsterite $[14,26]$, superior to those obtained with LDA or GGA type functionals. Iron, silicon and oxygen have been described by (8s)(64111sp)-(411d), (8s)-(6311sp)-(1d) and (8s)-(411sp)-(1d) contractions, respectively; these basis functions were already used by some of the present authors in previous works on $\mathrm{MgSiO}_{4}$ forsterite $[14,26]$ and $\mathrm{Fe}_{3} \mathrm{Al}_{2}\left(\mathrm{SiO}_{4}\right)_{3}$ almandine [18].

The level of accuracy in evaluating the Coulomb and Hartree-Fock exchange series is controlled by five parameters [22,27], for which the 777818 values were used. The threshold on the SCF energy was set to $10^{-10} \mathrm{Ha}$ for both the geometry optimisation and the frequency calculation. The reciprocal space was sampled according to a sublattice with shrinking factor [22] set to 6 (along the 3 lattice vectors), corresponding to 64 independent $\vec{k}$ vectors in the irreducible part of the Brillouin zone. 
The DFT exchange-correlation contribution is evaluated by numerical integration over the unit cell volume. In CRYSTAL, radial and angular points of the grid are generated through Gauss-Legendre radial quadrature and Lebedev two-dimensional angular point distributions. A $(75,974)$ p grid was used, corresponding to a pruned grid with 75 radial and 974 angular points (XLGRID keyword in the CRYSTAL09 manual [22]). The accuracy in the integration can be estimated by the error on the integrated electronic charge density in the unit cell $\left(\Delta_{e}=7 \cdot 10^{-6}|e|\right.$ on a total of 392 electrons).

The structure was optimized by using the analytical energy gradients with respect to atomic coordinates and unit-cell parameters [28, 29, 30], within a quasi-Newton scheme combined with the BFGS algorithm for Hessian updating [31, 32, 33, 34]. Convergence was checked on both gradient components and nuclear displacements, for which the default values [22] are chosen.

The calculation of the TO vibrational frequencies at the $\Gamma$ point has been performed within the harmonic approximation; the mass-weighted Hessian matrix W is constructed by numerical differentiation of the analytical gradients with respect to the atomic cartesian coordinates. The calculated (optimized) equilibrium geometry is taken as reference. Details on the calculation of vibrational frequencies can be found in Refs. 35 and 36.

The strength of the $n^{\text {th }}$ oscillator, $f_{n}$, is defined as:

$$
f_{n, i j}=\frac{4 \pi}{\Omega} \frac{\bar{Z}_{n, i} \bar{Z}_{n, j}}{v_{n}^{2}},
$$

where $\Omega$ is the unit cell volume, $i$ and $j$ indicate the three Cartesian components, $v_{n}$ is the frequency of the $n^{\text {th }}$ mode and

$$
\bar{Z}_{n, i}=\sum_{A, j} \mathbf{t}_{n, A j} Z_{A, i j}^{*} \frac{1}{\sqrt{M_{A}}} .
$$

$Z_{A, i j}^{*}$ is the Born effective charge tensor associated with atom $A$, evaluated through a Berry phase approach $[37,38,39] ; \mathbf{t}_{n, A i}$ is an element of the eigenvectors matrix $\mathrm{T}$ of the mass-weighted Hessian matrix $\mathrm{W}$, that transforms the cartesian atomic directions into the $n^{\text {th }}$ normal coordinate directions; $M_{A}$ is the mass of atom $A$.

The ionic components to the static dielectric tensor $\epsilon_{0, i j}$ are evaluated as the sum of the oscillator strengths: $F_{i j}=\sum_{n} f_{n, i j}$. The electronic high frequency components $\epsilon_{\infty, i j}$ are 
calculated through the Coupled-Perturbed KS/HF (Kohn-Sham/Hartree-Fock) scheme $[15,16,40,41,42]$.

Manipulation and visualization of structures have been performed with the Jmol 3D engine (jmol.sourceforge.net/; www.theochem.unito.it/crystal_tuto/mssc2008_cd/tutorials/webvib/index.html). Molecular drawings have been rendered with the Inkscape program (www.inkscape.org) using input files prepared with Jmol.

\subsection{The reflectance spectrum}

The reflectance spectrum $R(v)$ is defined as follows [43]:

$$
R(v)=\left|\frac{\sqrt{\epsilon(v)-\sin ^{2}(\theta)}-\cos (\theta)}{\sqrt{\epsilon(v)-\sin ^{2}(\theta)}+\cos (\theta)}\right|^{2},
$$

where $\theta$ is the incidence angle of the infrared beam; in the present work, we have set $\theta=10^{\circ}$, according to the experimental setup of our reference work [12].

In the case of orthorhombic systems, the complex dielectric function turns out to be a diagonal tensor, defined according to a classical damped harmonic oscillator model $[43,44]$ :

$$
\epsilon(v)_{i i}=\epsilon_{\infty, i i}+\sum_{n} \frac{f_{n, i i} v_{n}^{2}}{v_{n}^{2}-v^{2}-i v \gamma_{n}},
$$

where $v_{\mathrm{n}}, f_{n}$ and $\gamma_{\mathrm{n}}$ are the TO peak position, the oscillator strength and the damping factor. The electronic high frequency contributions $\epsilon_{\infty, i i}$ are almost constant with respect to frequency in the IR range, as electronic transition energies are very large compared to IR transition energies.

A microscopic approach to the calculation of the dielectric function permits to write $[44,45,46]$ :

$$
\epsilon(v)_{i i}=\epsilon_{\infty, i i}+\frac{4 \pi}{\Omega} \sum_{n} \frac{\bar{Z}_{n, i}^{2}}{v_{n}^{2}-v^{2}-i v \gamma_{n}},
$$

where the definition of the oscillator strength $f_{n, i i}$ according to eq. 1 has been used.

As the harmonic model is used in our simulation, we are unable to compute the $\gamma_{\mathrm{j}}$ damping factors. As a reasonable choice [17, 47, 48], all of them have been set equal to $9 \mathrm{~cm}^{-1}$, the $\bar{\gamma}^{\exp }$ mean value of the experimental $\gamma_{\mathrm{n}}^{\exp }$ data.

From the experimentalists' point of view [12], the digitalized $R^{\exp }(v)$ function, through eq. 3 and 4 , provides by best fit the "experimental" values for $v_{n}, f_{n}$ and $\gamma_{n}$. In 
the present case, 10, 10 and 7 experimental IR peaks were identified for the $x, y$, and $z$ directions, respectively; then the fitting function contains 30,30 and 21 parameters. The comparison among theory and experiment can be performed both at the $R(v)$ level, or by comparing calculated and measured $v_{\mathrm{n}}$ and $f_{n}$.

\section{Results}

\subsection{Structure}

The crystal structure of fayalite (see Figure 1) is built up by $\mathrm{SiO}_{4}$ and $\mathrm{FeO}_{6}$ distorted tetrahedra and octahedra, respectively. Si polyhedra share vertices with the Fe ones, but not with each other. There are two symmetry-independent Fe atoms, named Fe1 (on the inversion centre at $0,0,0)$ and $\mathrm{Fe} 2$ [4]. The Fe1 octahedra share edges forming rods parallel to the crystallographic $c$ axis, and the $\mathrm{Fe} 2$ octahedra are linked laterally to these rods by edge-sharing, too.

The calculated equilibrium geometry, given in Table 1, is in good agreement with experiment [4]. Cell parameters are slightly overestimated (largest deviation: $1.5 \%$ for a), as usual for B3LYP. Cell parameters computed by Ref. 19, also shown in Table 1, are largely overestimated $(a)$, in good agreement $(b)$ and slightly underestimated $(c)$. Compensation among these non systematic errors with respect to experiment leads to a fortuitous good agreement with the experimental volume.

The experimental $\mathrm{Si}-\mathrm{O}$ and $\mathrm{Fe}-\mathrm{O}$ distances are well reproduced (the largest difference is smaller than $0.04 \AA$ ). The Si tetrahedron turns out to be almost regular, in agreement with experiments. Larger distortions are shown by the Fe octahedra, as indicated by the cation-oxygen (Fe-O) distances, whose values span over $0.26 \AA$ ( $2.08 \div 2.34 \AA$ ); this is an evidence of the Jahn-Teller distortion of the octahedral sites. As a comparison, the range of $\mathrm{Mg}-\mathrm{O}$ distances in the case of the $\mathrm{Mg}$-rich olivine forsterite is restricted to $0.16 \AA$ [14].

\subsection{Electronic configuration}

Fayalite turns out to be quite ionic (see Table 2). Net charges $\mathrm{Q}$ obtained by a Mulliken population analysis are $+1.4|e|(\mathrm{Fe}$, the formal charge being +2$),+2.0|e|$ $(\mathrm{Si}),-1.2|e|(\mathrm{O})$. The $\mathrm{Fe}-\mathrm{O}$ bond population $\mathrm{B}$ is about $+0.05|e|$ (indicating ionic bond), whereas for the $\mathrm{Si}-\mathrm{O}$ bond it is five times larger $(+0.26|e|$, covalent bond $)$. 
The atomic integrated spin density $\mathrm{M}$ is given in Table 2, too. $\mathrm{M}$ is $\pm 3.8|e|$ (for " $\alpha$ " Fe and " $\beta$ " Fe, respectively), very close to the ideal value of a $\mathrm{d}^{6}$, high-spin configuration ( $\pm 4|e|$, resulting from $5 \alpha$ and $1 \beta$ electrons). The high-spin solution is well known to be more stable than the low-spin one in Fe-containing compounds at ambient conditions; extremely high pressures are required to induce the transition towards a low-spin state $[49,50,51]$. The spin maps given in Figure 2 show the lobe structure of the Fe density resulting from the orientation of the $6^{\text {th }}$ occupied d orbital that breaks the spherical symmetry of the $\mathrm{d}^{5}$ configuration.

Due to the presence of the Fe ion (in a $\mathrm{d}^{6}$, high-spin configuration), different spin states are possible; the present work concentrates on the most stable phase, namely an antiferromagnetic phase (four $\alpha$ polarized and four $\beta$ polarized Fe ions), where Fe planes along the $x$ axis show alternating spins (see Figure 1). This implies an antiferromagnetic order between ions at the centre of corner-sharing oxygen octahedra (Fe-O-Fe angle close to $180^{\circ}$ ) and a ferromagnetic order between edge-sharing octahedra $\left(\mathrm{Fe}-\mathrm{O}-\mathrm{Fe}\right.$ angle of about $\left.90^{\circ}\right)$. The resulting arrangement is consistent with a $\mathrm{Fe}-\mathrm{Fe}$ magnetic interaction via a superexchange mechanism through oxygen p orbitals [19]. The described antiferromagnetic phase is lower in energy than the ferromagnetic one (eight $\alpha$ polarized Fe ions) by $5.5 \mathrm{mHa}(14.4 \mathrm{~kJ} / \mathrm{mol})$ per unit cell.

A small spin polarization appears on oxygen atoms, which is responsible for the transmission of the magnetic information from one transition metal to the other, and for the energy difference among the stable antiferromagnetic (AFM) and the ferromagnetic (FM) phases. This mechanism is evident when comparing the FM (Figure 2(a)) and the AFM (Figure 2(b)) spin maps, showing the different oxygen polarization for the two cases; let us refer to the set of nearly collinear atoms Fe1 "A" - O - Fe2 "B". The spin density on $\mathrm{O}$ is higher in the FM case, where the presence of neighboring Fe ions with the same spin (say " $\alpha$ ") constrains the $\alpha$ electrons of $\mathrm{O}$ to pile up close to the atomic nucleus. In the AFM case, the opposite polarization of neighboring Fe ions permits $\mathrm{O}$ to displace $\alpha$ and $\beta$ electrons in opposite directions, with a consequent lower energy. The $\mathrm{M}$ values for the $\mathrm{O}$ atom under study are +0.10 and $+0.01|e|$, for the FM and AFM phases, respectively ("O3" oxygen in Table 2), documenting the reduced spin polarization on the $\mathrm{O}$ atoms in the AFM phase with respect to the FM one. 


\subsection{Infrared modes: wavenumbers and intensities}

Fayalite, as all olivines, is orthorhombic (space group Pbnm, n. 62). There are 28 atoms ( 4 formula units) in the unit-cell, 6 of which symmetry independent, giving rise to 84 vibrational modes. Symmetry analysis shows that:

$$
\Gamma_{\text {total }}=11 A_{g} \oplus 11 B_{1 g} \oplus 7 B_{2 g} \oplus 7 B_{3 g} \oplus 10 A_{u} \oplus 10 B_{1 u} \oplus 14 B_{2 u} \oplus 14 B_{3 u} .
$$

A total of 35 IR active modes $\left(9 B_{1 u} \oplus 13 B_{2 u} \oplus 13 B_{3 u}\right)$ and 36 Raman active modes $\left(11 A_{g} \oplus 11 B_{1 g} \oplus 7 B_{2 g} \oplus 7 B_{3 g}\right)$ is then expected, plus $10 A_{u}$ inactive modes. The three $B_{1 u}, B_{2 u}$ and $B_{3 u}$ modes left correspond to rigid translations.

In the present study we focus our attention on the 35 IR active modes, and compare the obtained results with the experimental ones by Suto and coworkers [12]. The experimental setup exploited a synchrotron radiation facility, providing high quality reflectance spectra.

In Tables 3, 4 and 5 the calculated and experimental wavenumbers and oscillator strengths are reported for the three crystallographic axes. The overall agreement between the two sets of data is very satisfactory: $\overline{|\Delta|}$ for the $a, b$ and $c$ axes is $4.2,4.3$ and $3.2 \mathrm{~cm}^{-1}$, respectively. $\bar{\Delta}$ is $4.0,4.3$ and $2.5 \mathrm{~cm}^{-1}$, indicating a small systematic overestimation of the calculated frequencies. $\left|\Delta_{\max }\right|$ is greater than $10 \mathrm{~cm}^{-1}$ only in three cases (one per crystal axis), and never exceeds $12 \mathrm{~cm}^{-1}$.

While 35 IR modes are predicted by our computation, only 27 peaks were observed experimentally. The missing peaks are equally distributed along the three directions: 3 (modes 1,6,9), 3 (modes 1,7,10) and 2 (modes 1,2) for the $a, b$ and $c$ axes, respectively. All these peaks correspond to modes with extremely low computed intensity (oscillator strength $f \leq 0.01$ ).

We begin our analysis of the oscillator strengths from the comparison of the sums $F^{e x p}=\sum_{j} f_{j}^{e x p}$ and $F^{c a l c}=\sum_{j} f_{j}^{c a l c}$. These quantities represent the ionic contribution to the static dielectric tensor (see Table 6 ); $F^{\text {calc }}$ is quite close to $F^{e x p}$, the differences being $-9.8 \%,-3.5 \%$ and $-1.5 \%$ for the $x, y$ and $z$ components (along the $a, b$ and $c$ axes, respectively).

When passing from $F$ to $\Delta F=\sum_{j}\left|\Delta f_{j}\right|$, that is the sum of the absolute differences (see Tables 3, 4 and 5), the situation is apparently less satisfactory, $\Delta F$ being $0.98,2.09$ and 
0.61 , that is $20.0 \%, 38.3 \%$ and $11.3 \%$ of the corresponding $F$.

A deeper analysis shows however that the largest contributions to $\Delta F$ are due to modes that are very close in frequency, (see lines grouped in columns 6,8 and 10 of Tables 3 and 4). The one-to-one correspondence between calculated and experimental peaks is arbitrary in these cases.

For this reason, $f$ belonging to two (or more) frequencies with $\Delta v \leq 15 \mathrm{~cm}^{-1}$, or with $\Delta v \leq 30 \mathrm{~cm}^{-1}$ and $f_{\text {calc }} \geq 0.5$, were grouped before comparison among theory and experiment. In this way we also eliminate a second source of discrepancy: when modes are close in frequency, the deconvolution process of the experimental spectrum can be affected by large errors, due to the strong correlation between the parameters describing the intensity of the two close peaks. This is evident in the couples of modes $(2,3)$ and $(4,5)$ of the $b$ axis, where the differences between experimental and calculated $f$ have opposite sign $(0.34,-0.29$ and $0.59,-0.74)$, and reduce to 0.05 and -0.15 if they are summed up before comparison.

This strategy reduces $\Delta F$ in the cases of the $a$ and $b$ axes to $0.66(13.5 \%)$ and 0.33 (6.1 $\%)$, respectively. Overall, also the agreement between experimental and calculated $f$ is then excellent.

\subsection{Infrared modes: isotopic substitutions}

Isotopic substitutions can be used to evaluate the participation of the various atoms to the vibrational modes in the different regions of the spectrum. In the present case, four different new spectra have been generated, in which ${ }^{56} \mathrm{Fe} 1$ was substituted by ${ }^{60} \mathrm{Fe} 1,{ }^{56} \mathrm{Fe} 2$ by ${ }^{60} \mathrm{Fe} 2,{ }^{28} \mathrm{Si}$ by ${ }^{30} \mathrm{Si}$ and ${ }^{16} \mathrm{O}$ by ${ }^{17} \mathrm{O}$. The shifts are represented graphically in Figure 4.

Fe1 shows large isotopic shifts $\left(2 \div 6 \mathrm{~cm}^{-1}\right)$ in a wide range of wavenumbers, $130 \div 360$ $\mathrm{cm}^{-1}$, whereas only four frequencies in the range $170 \div 260 \mathrm{~cm}^{-1}$ feature shifts larger than $2 \mathrm{~cm}^{-1}$ when the $\mathrm{Fe} 2$ mass is increased. This result is consistent with the Fe1 octahedra being tightly linked to one another by edge sharing, so as to form rigid [001] chains (see Figure 1), whereas the $\mathrm{Fe} 2$ octahedra are more loosely connected to the sides of these chains, and thus contribute to the lowest frequency modes only.

Silicon is mainly involved in the high frequency $\left(>850 \mathrm{~cm}^{-1}\right)$ stretching modes and in 
the bending modes around $460 \div 570 \mathrm{~cm}^{-1}$, with shifts up to 14 and $6 \mathrm{~cm}^{-1}$, respectively. As expected, oxygen is involved in all modes, with an isotopic shift which is roughly proportional to the mode frequency.

These results are closely similar to the ones presented for forsterite [14]. The main difference is due to the different mass of the cation in the octahedral position (Fe instead of $\mathrm{Mg}$ ): the involved modes extend up to $360 \mathrm{~cm}^{-1}$ here, and to $540 \mathrm{~cm}^{-1}$ in forsterite.

\subsection{Dielectric tensors}

The experimental values of the components of the high frequency electronic dielectric tensor $\epsilon_{\infty}$ are $3.55,3.35$ and 3.50 for the $a, b$ and $c$ axes, respectively (see Table 6), obtained through the best fit analysis of the reflectance curves. The calculated values, computed by means of a CPKS scheme [15, 16, 40, 41, 42], are 3.26, 3.02 and 3.25, $(-8.9 \%,-10.9 \%$ and $-7.7 \%$ with respect to experiment).

The static dielectric tensor $\epsilon_{0}$ is obtained as the sum of the electronic and ionic contributions (the latter have been discussed in Section 3.3). Computed components are $8.15,8.47$ and 8.65 , to be compared with the experimental ones, 8.92, 8.99 and 8.82 (the underestimation is $9.4 \%, 6.1 \%$ and $2.0 \%$, respectively).

\subsection{Reflectance curves}

The $R^{\text {calc }}(v)$ functions, computed from the frequencies and intensities obtained for the three axes, and damping factors set to $\bar{\gamma}^{\exp }=9 \mathrm{~cm}^{-1}$ (see Section 2.1), are shown in Figure 3 (black lines). In each panel, for comparison, both the instrumental $R^{\exp }(v)$ (gray thick line) and fitted $R^{f i t}(v)$ (black dotted line, constructed from the fitted parameters) experimental spectra are included [12].

The experimental spectra turn out to be reproduced extremely well by our simulated data for all the three axes, with the only exceptions of the doublets below $200 \mathrm{~cm}^{-1}$ in the case of $a$ and $b$ axes. This discrepancy, as other smaller discrepancies in amplitude along the three spectra, is mainly due to the use of a unique mean value $\bar{\gamma}^{\text {exp }}$ for all modes [17, 47, 48]. The root mean square RMS between the instrumental and the fitted/computed reflectance curves gives a quantitative indication of the quality of the spectra. In the case of $R^{f i t}$ the RMS values are $0.018,0.016$ and 0.013 for the $a$, $b$ and $c$ axes, respectively, while in the case of $R^{\text {calc }}$ the RMS values are $0.086,0.081$ 
and 0.081 . When comparing the three axes among them, the quality of the computed curves is very similar. It is worth noting that for all the axes $R^{\text {calc }}$ shows RMS values of the same order of magnitude as $R^{f i t}$.

\section{Conclusions}

The IR spectrum of fayalite was calculated by using ab initio techniques and compared to high quality experimental data. The comparison involves 27 wavenumbers and oscillator strengths, showing that:

(a) When high-quality experimental and calculated data are available, very good agreement can be achieved, and a very detailed description of vibrational properties (wavenumbers and oscillator strengths) can be provided;

(b) Factors that can reduce the accuracy of experiment (quality of the sample, experimental setup, accuracy of the deconvolution processes) or simulation (approximation of the Hamiltonian, finite basis set) are different, and a cross comparison can eliminate (or "filter") anomalous behaviours;

(c) Simulation integrates the experimental data, as it provides the complete set of infrared modes; in this respect, it confirms that a fraction of symmetry allowed modes does not appear in the experimental spectrum due to their low intensity;

(d) Simulation helps in the interpretation of the experimental spectrum, as it allows a detailed and objective analysis of the nature of the infrared modes: Fe- and $\mathrm{SiO}_{4}$ - related peaks are identified. 


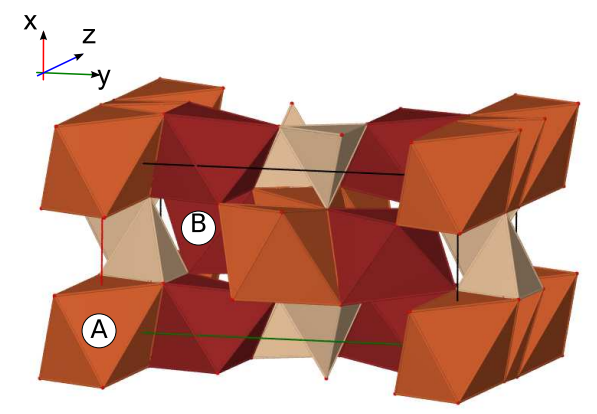

Figure 1: Perspective view of the crystal structure of fayalite, $\mathrm{Fe}_{2} \mathrm{SiO}_{4} . \mathrm{SiO}_{4}$ tetrahedra (light brown) and $\mathrm{FeO}_{6}$ octahedra corresponding to the two independent $\mathrm{Fe} 1$ (brown) and $\mathrm{Fe} 2$ (dark brown) atoms, are emphasized. $\mathrm{Fe} 1$ and $\mathrm{Fe} 2$ ions at the centre of the octahedra marked as "A" and "B", respectively, are represented in the spin density maps of Figure 2, too.

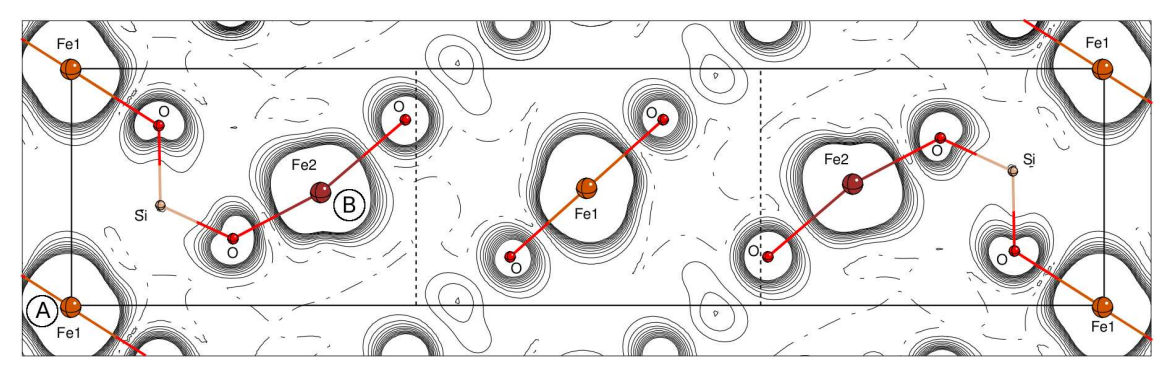

(a) FM (031) plane

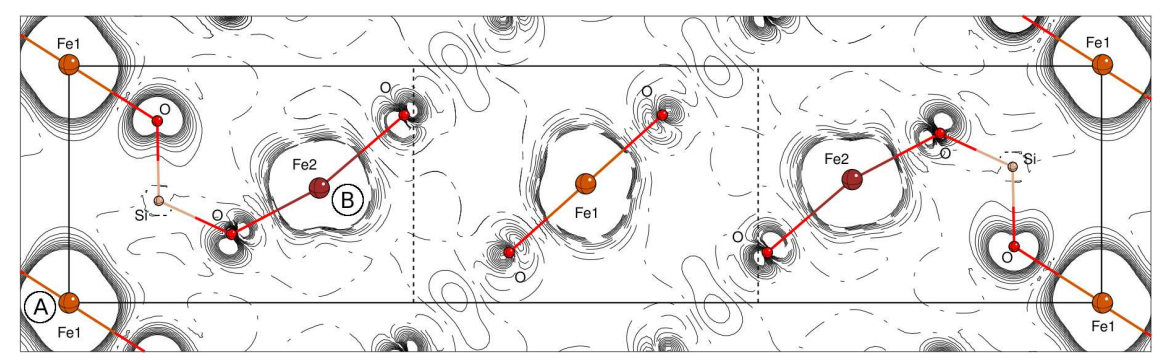

(b) AFM (031) plane

Figure 2: Spin density maps, on the (031) plane, of the Fe ions in the ferromagnetic (FM) and antiferromagnetic (AFM) phases of fayalite. For sake of clarity, Fe1 and Fe2 ions marked as "A" and "B", respectively, have been highlighted in Figure 1, too (see Section 3.2 for more details). Thick segments represent chemical bonds lying on the selected plane. 10 iso-density lines are reported, with $\rho_{\min }=0.01, \rho_{\max }=0.1$, $\delta \rho=0.01|e| / b o h r^{3}$. Continuous, dashed and dot-dashed isolines indicate positive, negative and null density, respectively. 


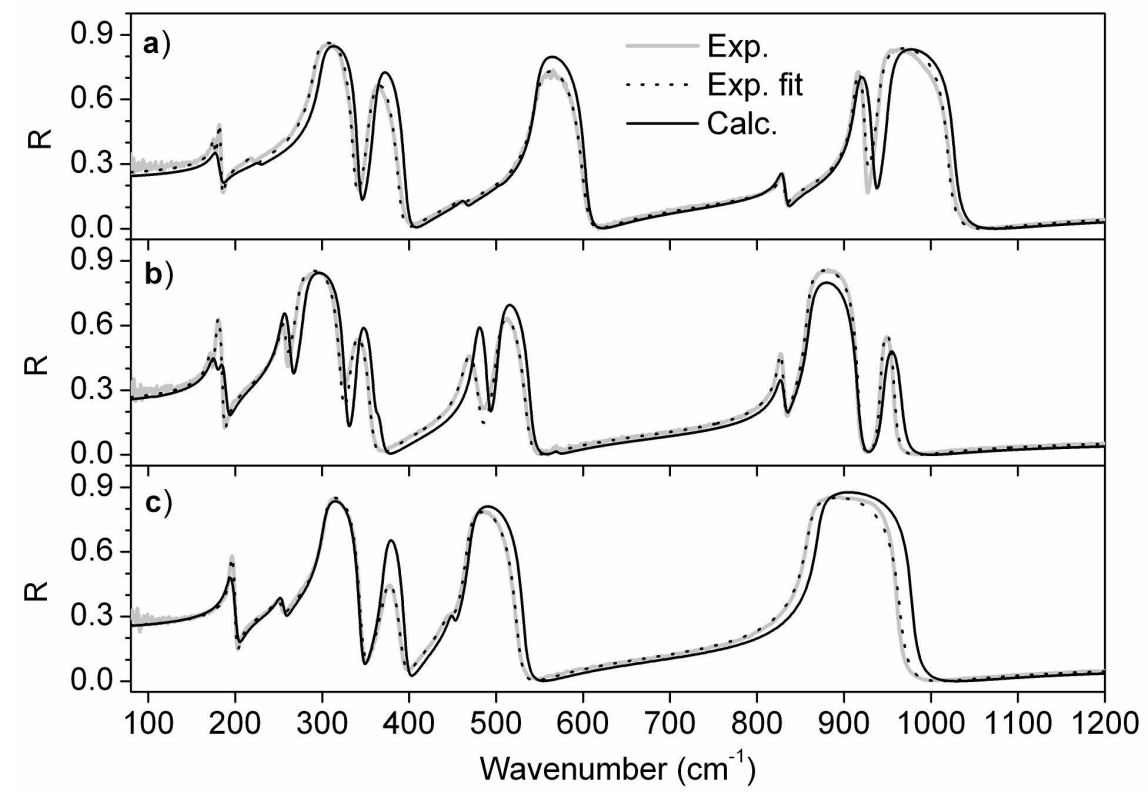

Figure 3: Calculated and experimental reflectance spectra for $a, b$ and $c$ axes of fayalite. Computed curves are obtained by using a damping factor equal to $9 \mathrm{~cm}^{-1}$. Experimental curves are a courtesy of Prof. Suto. Experimental fitted curves are taken from Ref. 12. 


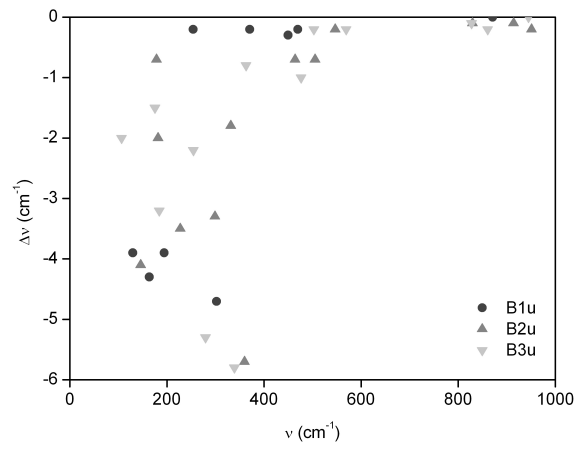

(a) ${ }^{56} \mathrm{Fe} 1$ substituted by ${ }^{60} \mathrm{Fe} 1$

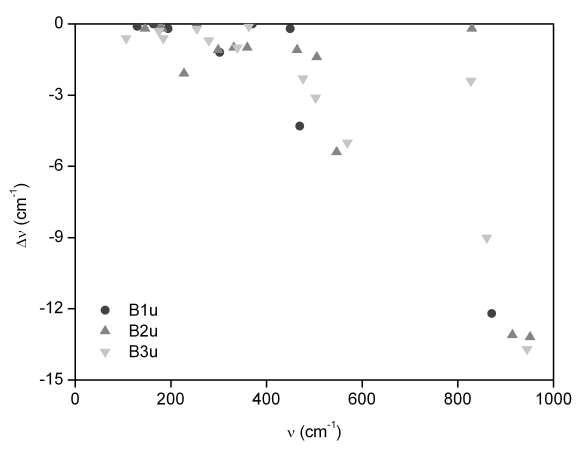

(c) ${ }^{28} \mathrm{Si}$ substituted by ${ }^{30} \mathrm{Si}$

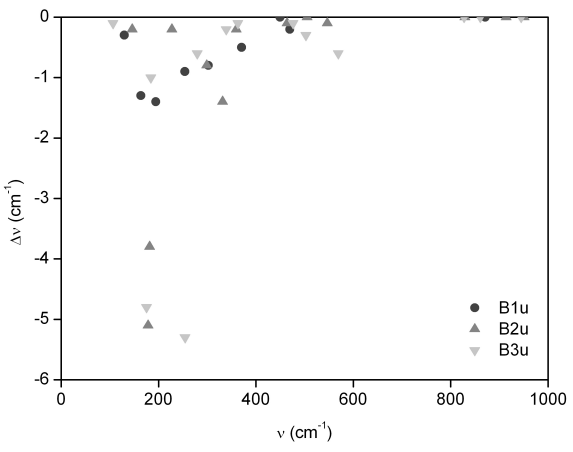

(b) ${ }^{56} \mathrm{Fe} 2$ substituted by ${ }^{60} \mathrm{Fe} 2$

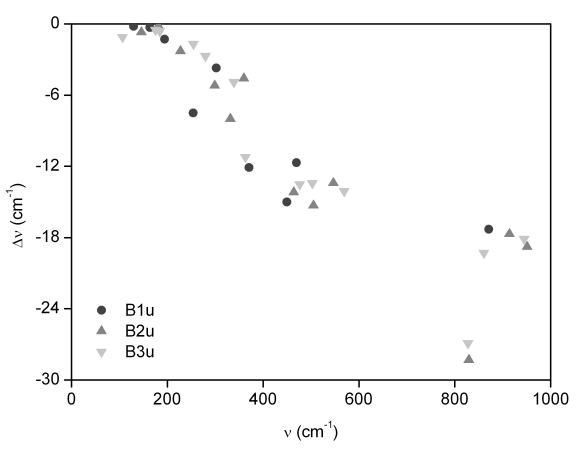

(d) ${ }^{16} \mathrm{O}$ substituted by ${ }^{17} \mathrm{O}$

Figure 4: Effect of the isotopic substitution in fayalite: isotopic shift $\left(\Delta v, \mathrm{~cm}^{-1}\right)$ for all the IR frequencies. 


\begin{tabular}{lccc}
\hline & Our work & Cococcioni $^{a}$ & Exp. $^{b}$ \\
\hline $\mathrm{a}$ & 4.8913 & 4.9531 & 4.822 \\
$\mathrm{~b}$ & 10.5684 & 10.4671 & 10.488 \\
$\mathrm{c}$ & 6.1565 & 5.9744 & 6.094 \\
\hline Vol. & 318.25 & 309.74 & 308.19 \\
\hline $\mathrm{Si}-\mathrm{O}_{\min }$ & 1.640 & 1.651 & 1.625 \\
$\mathrm{Si}-\mathrm{O}_{\text {max }}$ & 1.669 & 1.687 & 1.649 \\
$\mathrm{Fe} 1-\mathrm{O}_{\text {min }}$ & 2.144 & 2.153 & 2.121 \\
$\mathrm{Fe} 1-\mathrm{O}_{\text {max }}$ & 2.260 & 2.216 & 2.230 \\
$\mathrm{Fe} 2-\mathrm{O}_{\min }$ & 2.079 & 2.046 & 2.079 \\
$\mathrm{Fe} 2-\mathrm{O}_{\max }$ & 2.337 & 2.290 & 2.292 \\
\hline
\end{tabular}

Table 1: Calculated and experimental geometry of fayalite (Pbnm space group, 28 atoms in the unit cell, 6 of which symmetry independent). $a, b$ and $c$ are the cell parameters. The maximum (max) and minimum (min) $\mathrm{Si}-\mathrm{O}$ and Fe-O bond distances are reported. Distances are in $\AA$, volumes in $\AA^{3}$. ${ }^{a}$ Ref. $19 .{ }^{b}$ Ref. 4.

\begin{tabular}{l|lcccccc}
\hline & & $\mathrm{Fe} 1$ & $\mathrm{Fe} 2$ & $\mathrm{Si}$ & $\mathrm{O} 1$ & $\mathrm{O} 2$ & $\mathrm{O} 3$ \\
\hline & $\mathrm{Q}$ & +1.355 & +1.426 & +1.981 & -1.163 & -1.216 & -1.192 \\
$\mathrm{FM}$ & $\mathrm{B}$ & +0.042 & +0.054 & +0.259 & -0.020 & -0.034 & -0.034 \\
& $\mathrm{M}$ & +3.774 & +3.799 & +0.025 & +0.095 & +0.109 & +0.099 \\
& $\mathrm{~d}$ & 2.144 & 2.079 & 1.640 & 2.744 & 2.597 & 2.597 \\
\hline \multirow{4}{*}{$\mathrm{AFM}$} & $\mathrm{Q}$ & +1.352 & +1.422 & +1.980 & -1.163 & -1.213 & -1.189 \\
& $\mathrm{~B}$ & +0.042 & +0.055 & +0.259 & -0.020 & -0.034 & -0.034 \\
& $\mathrm{M}$ & \pm 3.763 & \pm 3.782 & \pm 0.002 & \pm 0.094 & $\mp 0.048$ & \pm 0.012 \\
& $\mathrm{~d}$ & 2.144 & 2.079 & 1.640 & 2.744 & 2.597 & 2.597 \\
\hline
\end{tabular}

Table 2: Mulliken population analysis of ferromagnetic (FM) and antiferromagnetic (AFM) phases of fayalite. Note that two and three symmetry independent atoms are present in the unit cell, in the case of Fe and $\mathrm{O}$ atomic species, respectively. $\mathrm{Q}$ is the net atomic charge, $\mathrm{B}$ the bond population (with respect to the closest oxygen atom), $\mathrm{M}$ the atomic spin moment; all these quantities are in $|e|$ units. $\mathrm{d}$ are the distances with respect to the closest oxygen atom (in $\AA$ ). Note that, in the AFM phase, lower symmetry results in a doubled set of independent atoms, featuring opposite $\mathrm{M}$ values. 


\begin{tabular}{|c|c|c|c|c|c|c|c|c|}
\hline \multicolumn{9}{|c|}{ Axis a } \\
\hline & $v_{\text {calc }}$ & $v_{\text {exp }}{ }^{a}$ & $\Delta v$ & $f_{\text {calc }}$ & & $f_{\text {exp }}{ }^{a}$ & $\Delta f$ & \\
\hline 1 & 146.0 & - & - & 0.00 & & - & - & \\
\hline 2 & 178.8 & 175.6 & 3.2 & 0.32 & & 0.16 & 0.16 & \\
\hline 3 & 181.6 & 181.5 & 0.1 & 0.07 & 0.39 & $0.30\} 0.46$ & -0.23 & -0.07 \\
\hline 4 & 227.6 & 220.5 & 7.1 & 0.04 & & 0.10 & -0.06 & \\
\hline 5 & 299.1 & 291.5 & 7.5 & 2.79 & & 3.10 & -0.31 & \\
\hline 6 & 332.0 & - & - & 0.00 & & - & - & \\
\hline 7 & 359.7 & 353.1 & 6.6 & 0.36 & & 0.35 & 0.01 & \\
\hline 8 & 464.2 & 460.8 & 3.3 & 0.02 & & 0.01 & 0.01 & \\
\hline 9 & 505.2 & - & - & 0.01 & & - & - & \\
\hline 10 & 546.7 & 545.6 & 1.2 & 0.68 & & 0.73 & -0.05 & \\
\hline 11 & 829.2 & 829.9 & -0.7 & 0.05 & & 0.03 & 0.02 & \\
\hline 12 & 914.1 & 913.2 & 0.9 & 0.37 & & 0.32 & 0.05 & \\
\hline 13 & 950.9 & 939.8 & 11.1 & 0.19 & & 0.27 & -0.08 & \\
\hline$F[\Delta F]$ & & & & 4.89 & & 5.37 & [0.98] & {$[0.66]$} \\
\hline$\overline{\mid \overline{\Delta \mid}}$ & & & 4.2 & & & & 0.10 & $(0.07)$ \\
\hline $\bar{\Delta}$ & & & 4.0 & & & & -0.05 & $(-0.05)$ \\
\hline$\left|\Delta_{\max }\right|$ & & & 11.1 & & & & 0.31 & $(0.31)$ \\
\hline$N$ & & & 10 & & & & 10 & (9) \\
\hline
\end{tabular}

Table 3: Calculated (B3LYP) and experimental IR active vibrational frequencies $v\left(\mathrm{~cm}^{-1}\right)$ and oscillator strengths $f$ (adimensional) along the $a$ axis ( $B_{2 u}$ symmetry) of fayalite. $\Delta v$ and $\Delta f$ are the differences between experimental and calculated quantities. $F=\sum_{j} f_{j}$ is the sum of the oscillator strengths, $\Delta F=$ $\sum_{j}\left|\Delta f_{j}\right|$ is the sum of the absolute differences between experimental and calculated oscillator strengths. Statistical indices ( $x$ is either $v$ or $f$ ): $\overline{|\Delta|}=\sum_{i=1}^{N}\left|\Delta x_{i}\right| / N$ is the mean absolute difference, $\bar{\Delta}=\sum_{i=1}^{N} \Delta x_{i} / N$ is the mean difference, $\left|\Delta_{\max }\right|$ is the maximum difference, $N$ is the number of available experimental data, on which statistics are performed. Oscillator strengths are grouped either when the corresponding calculated frequencies differ $(\delta v)$ by less than $15 \mathrm{~cm}^{-1}$, or when $\delta v \leq 30 \mathrm{~cm}^{-1}$ and $f_{\text {calc }} \geq 0.5$. ${ }^{a}$ Ref. 12 . 


\begin{tabular}{|c|c|c|c|c|c|c|c|c|c|}
\hline \multicolumn{10}{|c|}{ Axis b } \\
\hline & $v_{\text {calc }}$ & $v_{e x p}{ }^{a}$ & $\Delta v$ & $f_{\text {calc }}$ & & $f_{\text {exp }}{ }^{a}$ & & $\Delta f$ & \\
\hline 1 & 106.7 & - & - & 0.01 & & - & & - & \\
\hline 2 & 175.5 & 172.0 & 3.5 & 0.64 & & 0.30 & & 0.34 & \\
\hline 3 & 184.5 & 179.1 & 5.3 & 0.50 & 1.14 & 0.79 & 1.09 & -0.29 & 0.05 \\
\hline 4 & 254.5 & 254.5 & 0.0 & 1.57 & & 0.98 & & 0.59 & \\
\hline 5 & 279.4 & 272.0 & 7.4 & 1.51 & 3.08 & 2.25 & 3.23 & -0.74 & -0.15 \\
\hline 6 & 339.1 & 332.1 & 7.0 & 0.14 & & 0.14 & & 0.00 & \\
\hline 7 & 363.2 & - & - & 0.01 & & - & & - & \\
\hline 8 & 476.5 & 465.5 & 11.0 & 0.40 & & 0.40 & & 0.00 & \\
\hline 9 & 502.5 & 500.5 & 2.0 & 0.18 & & 0.24 & & -0.06 & \\
\hline 10 & 569.6 & - & - & 0.01 & & - & & - & \\
\hline 11 & 827.7 & 826.4 & 1.2 & 0.09 & & 0.12 & & -0.03 & \\
\hline 12 & 860.6 & 858.4 & 2.2 & 0.36 & & 0.39 & & -0.03 & \\
\hline 13 & 945.0 & 941.6 & 3.4 & 0.04 & & 0.03 & & 0.01 & \\
\hline$F[\Delta F]$ & & & & 5.45 & & 5.64 & & {$[2.09]$} & {$[0.33]$} \\
\hline$\overline{|\Delta|}$ & & & 4.3 & & & & & 0.21 & $(0.04)$ \\
\hline $\bar{\Delta}$ & & & 4.3 & & & & & -0.02 & $(-0.03)$ \\
\hline$\left|\Delta_{\max }\right|$ & & & 11.0 & & & & & 0.74 & $(0.15)$ \\
\hline$N$ & & & 10 & & & & & 10 & (8) \\
\hline
\end{tabular}

Table 4: Calculated and experimental IR active vibrational frequencies and oscillator strengths along the $b$ axis $\left(B_{3 u}\right.$ symmetry). Symbols and units as in Table 3. ${ }^{a}$ Ref. 12.

\begin{tabular}{|c|c|c|c|c|c|c|}
\hline \multicolumn{7}{|c|}{ Axis c } \\
\hline & $v_{\text {calc }}$ & $v_{\exp }{ }^{a}$ & $\Delta v$ & $f_{\text {calc }}$ & $f_{\text {exp }}{ }^{a}$ & $\Delta f$ \\
\hline 1 & 130.0 & - & - & 0.00 & - & - \\
\hline 2 & 163.9 & - & - & 0.00 & - & - \\
\hline 3 & 194.1 & 195.4 & -1.3 & 0.85 & 0.63 & 0.22 \\
\hline 4 & 253.7 & 251.4 & 2.4 & 0.27 & 0.20 & 0.07 \\
\hline 5 & 302.0 & 302.7 & -0.7 & 2.45 & 2.58 & -0.13 \\
\hline 6 & 370.7 & 371.2 & -0.5 & 0.41 & 0.37 & 0.04 \\
\hline 7 & 449.6 & 447.0 & 2.6 & 0.08 & 0.16 & -0.08 \\
\hline 8 & 469.8 & 466.4 & 3.4 & 0.66 & 0.64 & 0.02 \\
\hline 9 & 870.9 & 859.1 & 11.8 & 0.68 & 0.74 & -0.06 \\
\hline$F[\Delta F]$ & & & & 5.40 & 5.32 & [0.61] \\
\hline$\overline{\overline{|\Delta|}}$ & & & 3.2 & & & 0.09 \\
\hline $\bar{\Delta}$ & & & 2.5 & & & 0.01 \\
\hline$\left|\Delta_{\max }\right|$ & & & 11.8 & & & 0.22 \\
\hline$N$ & & & 7 & & & 7 \\
\hline
\end{tabular}

Table 5: Calculated and experimental IR active vibrational frequencies and oscillator strengths along the $c$ axis $\left(B_{1 u}\right.$ symmetry). Symbols and units as in Table 3. ${ }^{a}$ Ref. 12.

\begin{tabular}{c|cc|cc|cc}
\hline & \multicolumn{2}{|c|}{$\epsilon_{0}$} & \multicolumn{2}{c|}{$\epsilon_{\infty}$} & \multicolumn{2}{c}{$F$} \\
& Calc. & Exp. $^{a}$ & Calc. & Exp. $^{a}$ & Calc. & Exp. $^{a}$ \\
\hline$x$ & 8.15 & 8.92 & 3.26 & 3.55 & 4.89 & 5.37 \\
$y$ & 8.47 & 8.99 & 3.02 & 3.35 & 5.45 & 5.64 \\
$z$ & 8.65 & 8.82 & 3.25 & 3.50 & 5.40 & 5.32 \\
\hline
\end{tabular}

Table 6: Calculated and experimental static dielectric tensor $\left(\epsilon_{0}\right)$ and its components: the electronic high frequency $\left(\epsilon_{\infty}\right)$ and the ionic one, evaluated as the sum of the oscillator strengths $\left(F=\sum_{j} f_{j}\right)$. ${ }^{a}$ Ref. 12 ; experimental values obtained through a fitting procedure. The three cartesian directions correspond to the crystallographic ones, so that the dielectric tensor turns out to be diagonal. 
[1] Deer, W.; Howie, R.; Zussman, J. An introduction to the rock forming minerals; John Wiley: New York, 1992.

[2] Molster, F. J.; Waters, L. B. F. M.; Trams, N. R.; van Winckel, H.; Decin, L.; van Loon, J. T.; Jager, C.; Henning, T.; Kaufl, H. U.; De Koter, A.; Bouwman, J. Astronomy and Astrophysics 1999, 350, 163-180.

[3] Smyth, J. R. American Mineralogist 1975, 60, 1092-1097.

[4] Kudoh, Y.; Takeda, H. Physica B+C 1986, 139, 333-336.

[5] Hofmeister, A. M.; Pitman, K. M. Physics and Chemistry of Minerals 2007, 34, 319-333.

[6] Dachs, E.; Geiger, C.; von Seckendorff, V.; Grodzicki, M. The Journal of Chemical Thermodynamics 2007, 39, 906-933.

[7] Hofmeister, A. M. Physics and Chemistry of Minerals 1997, 24, 535-546.

[8] Jager, C.; Molster, F. J.; Dorschner, J.; Henning, T.; Mutschke, H.; Waters, L. B. F. M. Astronomy and Astrophysics 1998, 339, 904-916.

[9] Koike, C.; Shibai, H.; Tuchiyama, A. Monthly Notices of the Royal Astronomical Society 1993, 264, 654-658.

[10] Koike, C.; Tsuchiyama, A.; Shibai, H.; Suto, H.; Tanabe, T.; Chihara, H.; Sogawa, H.; Mouri, H.; Okada, K. Astronomy and Astrophysics 2000, 363, 1115-1122.

[11] Koike, C.; Chihara, H.; Tsuchiyama, A.; Suto, H.; Sogawa, H.; Okuda, H. Astronomy and Astrophysics 2003, 399, 1101-1107.

[12] Suto, H.; Koike, C.; Sogawa, H.; Tsuchiyama, A.; Chihara, H.; Mizutani, K. Astronomy and Astrophysics 2002, 389, 568-571.

[13] Pitman, K. M.; Dijkstra, C.; Hofmeister, A. M.; Speck, A. K. Monthly Notices of the Royal Astronomical Society 2010, 406, 460-481.

[14] Noël, Y.; Catti, M.; D’Arco, P.; Dovesi, R. Physics and Chemistry of Minerals 2006, 33, 383-393. 
[15] Ferrero, M.; Rérat, M.; Orlando, R.; Dovesi, R. Journal of Computational Chemistry 2008, 29, 1450-1459.

[16] Ferrero, M.; Rérat, M.; Orlando, R.; Dovesi, R. Journal of Chemical Physics 2008, $128,014110$.

[17] Maschio, L.; Ferrabone, M.; Meyer, A.; Garza, J.; Dovesi, R. Chemical Physics Letters 2011, 501, 612-618.

[18] Ferrari, A. M.; Valenzano, L.; Meyer, A.; Orlando, R.; Dovesi, R. Journal of Physical Chemistry A 2009, 113, 11289-11294.

[19] Cococcioni, M.; Dal Corso, A.; de Gironcoli, S. Physical Review B 2003, 67, 094106.

[20] Jiang, X.; Guo, G. Physical Review B 2004, 69, 155108.

[21] Stackhouse, S.; Stixrude, L.; Karki, B. B. Earth and Planetary Science Letters 2010, 289, 449-456.

[22] Dovesi, R.; Saunders, V. R.; Roetti, C.; Orlando, R.; Zicovich-Wilson, C. M.; Pascale, F.; Civalleri, B.; Doll, K.; Harrison, N. M.; Bush, I. J.; D’Arco, P.; Llunell, M. CRYSTAL 2009 User's Manual; University of Torino, Torino, 2009.

[23] Becke, A. D. Journal of Chemical Physics 1993, 98, 5648-5652.

[24] Lee, C.; Yang, W.; Parr, R. G. Physical Review B 1988, 37, 785-789.

[25] Stephens, P. J.; Devlin, F. J.; Chabalowski, C. F.; Frisch, M. J. Journal of Physical Chemistry 1994, 98, 11623-11627.

[26] Demichelis, R.; Civalleri, B.; Ferrabone, M.; Dovesi, R. International Journal of Quantum Chemistry 2010, 110, 406-415.

[27] Pisani, C.; Dovesi, R.; Roetti, C.; Causà, M.; Orlando, R.; Casassa, S.; Saunders, V. R. International Journal of Quantum Chemistry 2000, 77, 1032-1048.

[28] Doll, K. Computational Physics Communications 2001, 137, 74-88. 
[29] Doll, K.; Harrison, N. M.; Saunders, V. R. International Journal of Quantum Chemistry 2001, 82, 1-13.

[30] Civalleri, B.; D’Arco, P.; Orlando, R.; Saunders, V. R.; Dovesi, R. Chemical Physics Letters 2001, 348, 131-138.

[31] Broyden, C. G. Journal of the Institute of Mathematics and its Applications 1970, $6,76-90$.

[32] Fletcher, R. Computer Journal 1970, 13, 317-322.

[33] Goldfarb, D. Mathematics of Computation 1970, 24, 23-26.

[34] Shanno, D. F. Mathematics of Computation 1970, 24, 647-656.

[35] Pascale, F.; Zicovich-Wilson, C. M.; Gejo, F. L.; Civalleri, B.; Orlando, R.; Dovesi, R. Journal of Computational Chemistry 2004, 25, 888-897.

[36] Zicovich-Wilson, C. M.; Pascale, F.; Roetti, C.; Orlando, R.; Saunders, V. R.; Dovesi, R. Journal of Computational Chemistry 2004, 25, 1873-1881.

[37] Dall'Olio, S.; Dovesi, R.; Resta, R. Physical Review B 1997, 56, 10105-10114.

[38] Baranek, P.; Zicovich-Wilson, C. M.; Roetti, C.; Orlando, R.; Dovesi, R. Physical Review B 2001, 64, 125102.

[39] Noël, Y.; Zicovich-Wilson, C. M.; Civalleri, B.; D’Arco, P.; Dovesi, R. Physical Review B 2002, 65, 014111.

[40] Ferrero, M.; Rérat, M.; Kirtman, B.; Dovesi, R. Journal of Chemical Physics 2008, 129, 244110 .

[41] Ferrero, M.; Rérat, M.; Orlando, R.; Dovesi, R. AIP Conf. Proc. 2007, 963, 1199_ 1203.

[42] Ferrero, M.; Civalleri, B.; Rérat, M.; Orlando, R.; Dovesi, R. Journal of Chemical Physics 2009, 131, 214704. 
[43] Decius, J. C.; Hexter, R. M. Molecular vibrations in crystals; McGraw-Hill: New York, 1977.

[44] Born, M.; Huang, K. Dynamical theory of crystal lattices; Oxford University Press: Oxford, 1954.

[45] Gonze, X.; Lee, C. Physical Review B 1997, 55, 10355-10368.

[46] Kleinmann, D. A.; Spitzer, W. G. Physical Review 1962, 125, 16-30.

[47] Dovesi, R.; De La Pierre, M.; Ferrari, A. M.; Pascale, F.; Maschio, L.; ZicovichWilson, C. M. accepted for publication in American Mineralogist, 2011.

[48] De La Pierre, M.; Orlando, R.; Maschio, L.; Doll, K.; Ugliengo, P.; Dovesi, R. Journal of Computational Chemistry 2011, 32, 1775-1784.

[49] Badro, J.; Rueff, J. P.; Vanko, G.; Monaco, G.; Fiquet, G.; Guyot, F. Science 2004, $305,383-386$.

[50] Tsuchiya, T.; Wentzcovitch, R. M.; da Silva, C. R. S.; de Gironcoli, S. Physical Review Letters 2006, 96, 198501.

[51] Rouquette, J.; Kantor, I.; McCammon, C. A.; Dmitriev, V.; Dubrovinsky, L. S. Inorganic Chemistry 2008, 47, 2668-2673. 